\title{
Reduced Complexity by Combining Norm Based Ordering MMSE-BSIDE Detection in MIMO Systems
}

\author{
Ramya Jothikumar and Nakkeeran Rangaswamy
}

\begin{abstract}
The breadth first signal decoder (BSIDE) is well known for its optimal maximum likelihood (ML) performance with lesser complexity. In this paper, we analyze a multiple-input multiple-output (MIMO) detection scheme that combines; column norm based ordering minimum mean square error (MMSE) and BSIDE detection methods. The investigation is carried out with a breadth first tree traversal technique, where the computational complexity encountered at the lower layers of the tree is high. This can be eliminated by carrying detection in the lower half of the tree structure using MMSE and upper half using BSIDE, after rearranging the column of the channel using norm calculation. The simulation results show that this approach achieves $22 \%$ of complexity reduction for $2 \times 2$ and $50 \%$ for $4 \times 4$ MIMO systems without any degradation in the performance.
\end{abstract}

Keywords-MIMO, MMSE, SD, ML, BSIDE, breadth first search.

\section{INTRODUCTION}

Multiple-input multiple-output (MIMO) has been recognized as a promising technology that provides a large spectral efficiency for wireless services [1]. Spatial multiplexing MIMO wireless system offers higher transmission rate without demanding extra spectra. The major goal of the spatially multiplexed MIMO detector is to extract the information bearing signal by mitigating the inter symbol interference and noise effects. In order to attain this, a number of decoding algorithm with different complexity-performance tradeoff emerges in the literature. For instance, the maximum likelihood (ML) detection achieves minimum error probability and hence considered to be the optimal detection method [2]. The computational complexity in realizing the ML metrics are prohibitively high which increases with the number of antennas and order of modulations, making it unfeasible for real time implementation. Sphere decoder (SD) emerges as a suboptimal decoder capable of solving the ML detection problem with less complexity. Although SD [3] - [6] meets out the ML performance, it still requires considerable computation. The drawback of SD lies in the estimation of the SNR to determine the initial radius of the sphere, which results in variable throughput. Though K-best decoder results in constant throughput with implementation possible, but the complexity reduction came at the cost of performance degradation. Many

R. Jothikumar is with the Dept of Electronics and Communication Engineering, Sri Manakula Vinayagar Engineering College, India (e-mail: mithulramya@ rediffmail.com).

N. Rangaswamy is with the Department of Electronics Engineering, School of Engineering and Technology, Pondicherry University, India (e-mail: nakkeeranpu@gmail.com). variants of $\mathrm{SD}$, such as probabilistic tree pruning, radius tightening [7] - [9], fixed sphere decoding has been proposed. The linear detection methods such as zero forcing (ZF) or minimum mean square error (MMSE) have the advantage of being less complex but provide suboptimal performance. The degradation in performance is managed by ordered successive interference cancellation (OSIC) techniques to some extend with slightly higher complexity than ZF and MMSE [10]-[12]. Motivated by the reliable potential of MMSE in detecting high SINR symbols with less complexity, a hybrid scheme that combines norm based ordering MMSE and breadth first signal decoder (BSIDE) algorithm is analyzed.

\section{MIMO SYSTEM MODEL}

MIMO systems with $\mathrm{N}_{T}$ transmit antennas and $\mathrm{N}_{R}$ receive antennas as shown in Fig. 1 is considered. The received signal model is given by

$$
\bar{Y}=\bar{H} \bar{S}+\bar{v}
$$

Where $\bar{Y}$ denotes the $\mathrm{N}_{\mathrm{R}} \mathrm{x} 1$ received complex symbol vector. $\bar{S}$ denotes the $\mathrm{N}_{\mathrm{T} X} 1$ transmitted complex symbol vector that contains entries from QAM constellation. $\mathrm{H}$ denotes $\mathrm{N}_{\mathrm{R}} \mathrm{X} \mathrm{N}_{\mathrm{T}}$ matrix of independent and identically distributed (i.i.d) Gaussian random noise components.
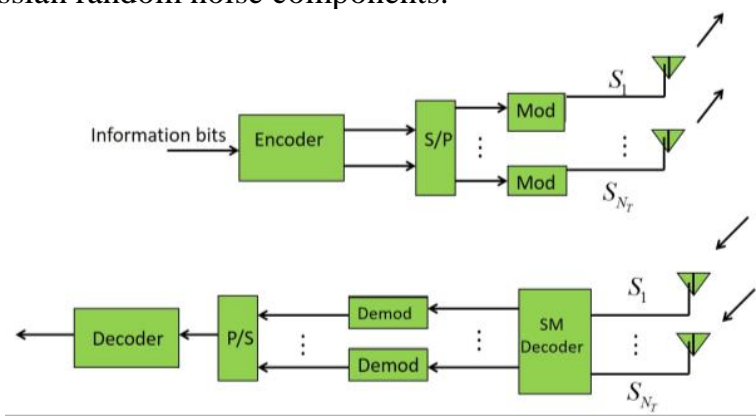

Fig. 1.Representation of Spatial Multiplexing MIMO systems

The real value decomposed $S$ vector contains $\mathrm{M}=2 \mathrm{~N}_{\mathrm{T}}$ elements and the $\mathrm{Y}$ vector contains $\mathrm{N}=2 \mathrm{~N}_{\mathrm{R}}$ elements (i.e ${ }^{\bar{H}}$ is $\mathrm{N} \times \mathrm{M}$ ). The ML detection of the transmitted symbol vector is given by

$$
\hat{S}=\arg _{S \in \Omega^{M}}^{\min }\|Y-H S\|^{2}
$$

Where $\Omega$ is the set, containing all possible modulation points. After QR decomposition, the equation (1) can be written as 


$$
\begin{aligned}
\|Y-H S\|^{2} & =\|Y-Q R S\|^{2} \\
& =\left\|Q^{H} Y-R S\right\|^{2} \\
= & \sum_{i=1}^{M}\left\|\hat{Y}_{i}-\sum_{j=i}^{M} R_{i, j} S_{j}\right\|^{2}
\end{aligned}
$$

Where $\hat{Y}_{i}$ represents the $\mathrm{i}^{\text {th }}$ element of vector $\hat{Y}=Q^{H} \boldsymbol{Y}$, $\boldsymbol{R}_{i, j}$ denotes the (i,j) ${ }^{\text {th }}$ element of the matrix $\mathrm{R}$ and $\mathrm{S}_{\mathrm{j}}$ is the $\mathrm{j}^{\text {th }}$ element of vector $\mathrm{S}$.

\section{EXISTING ALGORITHM}

A similar tree structure as shown in Fig.2, illustrates how the existing algorithm [14] works. In the tree, there are $\mathrm{N}$ layers with C-ary. Let $S_{l}^{(b)}=\left[S_{1, l}^{(b)}, S_{2, l}^{(b)}, \ldots \ldots, S_{N-l+1, l}^{(b)}\right]^{T}$ be a vector at the $\mathrm{b}^{\text {th }}$ node in $l^{\text {th }}$ layer of the tree, where $\mathbf{1} \leq \boldsymbol{b} \leq \boldsymbol{C}^{N-l+1}$ and $\mathbf{1} \leq \boldsymbol{l} \leq N$. The breadth first search strategy is followed, where the decision for each layer is made at that appropriate layer. The node distance for each node at the $\mathrm{N}^{\text {th }}$ layer is computed. The node with distance larger than $\mathrm{d}_{\mathrm{N}}$ is discarded. Next, all the node distances at the $(\mathrm{N}-1)^{\mathrm{th}}$ layer resulting from the undiscarded node at the previous layer is computed. The node with distance larger than $\mathrm{d}_{\mathrm{N}^{-}}-1$ is discarded. This procedure is repeated until layer 1 , to get the ML solution. To get the parameter $d_{1}$ smaller, BSIDE, estimate the ML solution using DFE detection algorithm at the beginning.

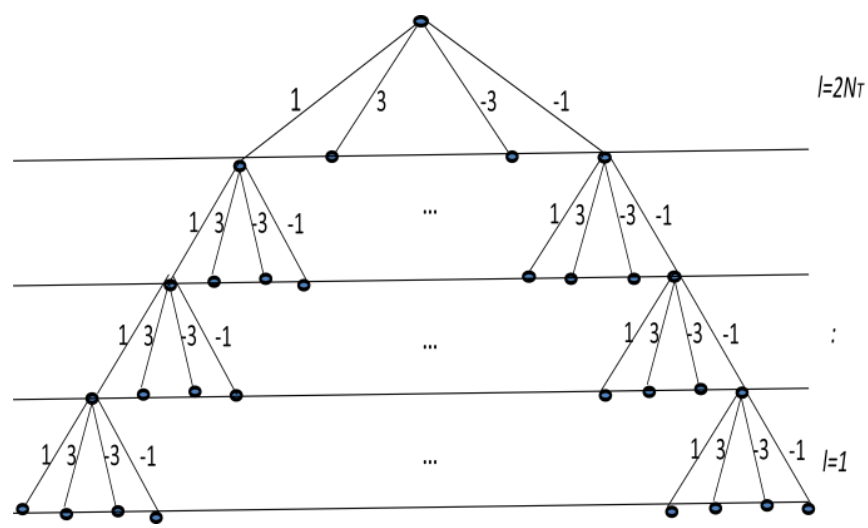

Fig. 2.Tree representation of 2×2 16- QAM MIMO systems

Let $\tilde{S}=\left[\tilde{S}_{1}, \tilde{S}_{2} \ldots \ldots . \tilde{S}_{N}\right]^{T}$ and $\tilde{d}=\|Y-R \tilde{S}\|^{2}$ be the decision feedback equalization (DFE) solution and distance respectively. The parameter $\mathrm{d}_{1}$ can be determined by

$$
d_{l}=\min \left\{d_{l+1},\left\|Y-R a^{(l)}\right\|^{2}\right\} \quad l=N, N-1 \ldots .2
$$

with $d_{N+1}=\tilde{d}_{\text {where }}$

$$
a^{(l)}=\left[\tilde{S}_{1}, \tilde{S}_{2}, \ldots \ldots \ldots, \tilde{S}_{l-1}, S_{1, l}^{[1]}, S_{2, l}^{[1]}, \ldots \ldots, S_{N-l+1, l}^{[1]}\right]^{T}
$$

Let $e_{l}$ be the undiscarded node at the $l^{\text {th }}$ layer with $e_{l}+1=1$. Then the node distance at the $l^{\text {th }}$ layer for $\left\{\Phi\left(\hat{S}_{l}^{(b)}\right)\right\}_{b=1}^{c e_{l+1}}$ is given as

$$
\begin{aligned}
\Phi\left[S_{1, N}^{(j)}, S_{1, l+1}^{(q)} \ldots . . S_{N-l, l+1}^{(q)}\right]^{T}= & \left(\hat{Y}_{l}-R_{l, l} S_{1, N}^{(j)}-\sum_{i=l+1}^{N} R_{l, i} S_{i-l, l+1}^{(q)}\right)^{2}+\ldots \\
& \sum_{j=l+1}^{N}\left(\hat{Y}_{j}-\sum_{i=l+1}^{N} R_{j, i} S_{i-l, l+1}^{(q)}\right)^{2} \\
= & \left(\hat{Y}_{l}-R_{l, l} S_{1, N}^{(j)}-\Theta\left(S_{l+1}^{(q)}\right)\right)^{2}+\Phi\left(S_{l+1}^{(q)}\right)
\end{aligned}
$$

for $j=1,2 \ldots . C$ and $q=1,2 \ldots . . e_{l+1}$.

\section{PROPOSED ALGORITHM}

BSIDE is a breadth first search algorithm having same bit error rate performance as the conventional ML decoder while allowing significant lower computational complexity. Since BSIDE is a combinational algorithm that shifts between DFE and ML, it performs well for signals with high and low SNR. Such that, if the SNR is low, ML detection is carried out and for symbols with high SNR, DFE is adopted without estimating the SNR of the signal due to its intrinsic nature. One drawback of BSIDE is that, in the case, if the signal to be detected false in the intermediate region of SNR, automatically the detection is carried out with ML estimation which generally requires high computational complexity. To overcome this, and to reduce the computational complexity of BSIDE, a hybrid MIMO detection scheme is developed.

This scheme combines the BSIDE algorithm with norm based ordering MMSE, in which for signals, with intermediate SNR, the direct adoption of MMSE as part of the final solution can reduce the search space, without the need for estimation of SNR. The investigation is carried out with the breadth first tree traversal technique, where the complexity encountered at the lower layers of the tree is high. This can be eliminated by carrying detection in the lower half of the tree structure using MMSE and upper half using BSIDE, after rearranging the column of the channel using norm calculation. A tree representation is very helpful in illustrating how the proposed scheme works as shown in Fig. 3. The proposed analysis is explained with the following step sequences as follows

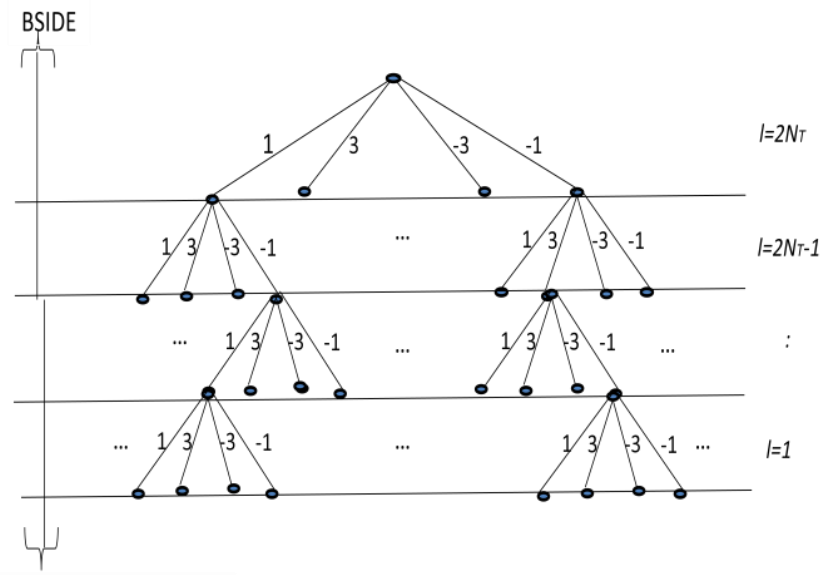

Norm based ordering MMSE

Fig. 3.Tree representation of 2x2 16- QAM MIMO systems that illustrates the STEP 1 combination of MMSE and BSIDE

Since the received signal strength of the transmitted signal is proportional to the norm of the appropriate column in the 
channel matrix, detection can be carried out in the order of the matrix. The norm of the column vectors in a channel matrix is calculated and arranged in descending order. The MMSE detected symbol vector $\tilde{S}_{M M S E}=\left[\tilde{S}_{M M S E}^{1} \ldots \ldots . . \tilde{S}_{M M S E}^{N_{T}}\right]^{T}$ is obtained. Let the permuted $\mathrm{H}$ matrix be $\tilde{H}$.

STEP 2

Perform QR decomposition of $\tilde{H}$ and calculate $\tilde{d}=\|Y-R \tilde{S}\|^{2}$ and let $l=N, \tilde{d}=d_{l+1}, t v=\tilde{S}$

\section{STEP 3}

Compute all node distances at the $\mathrm{N}^{\text {th }}$ layer and obtain $S_{N}^{[1]}$. If $\left[t v_{1}, t v_{l+1}, \ldots . t v_{N}\right]^{T}=S_{l}^{[1]}$,let $d_{l}=d_{l+1}$, and if $\left[t v_{1}, t v_{l+1}, \ldots . t v_{N}\right]^{T} \neq S_{l}^{[1]}$ and $l \leq \frac{N}{2}$, update symbol with appropriate MMSE entries.

\section{STEP 4}

If $\left[t v_{1}, t v_{l+1}, \ldots . t v_{N}\right]^{T} \neq S_{l}^{[1]}$ and $\quad l>\frac{N}{2}$, compute $\left\|Y-R a^{(l)}\right\|^{2}$. If $\left\|Y-R a^{(l)}\right\|^{2} \geq d_{l+1}$ let $d_{l}=d_{l+1}$. Else if $\left\|Y-R a^{(l)}\right\|^{2}<d_{l+1}$ let $d_{l}=\left\|Y-R a^{(l)}\right\|^{2}$ and $t v=a^{(l)}$.

It is worthwhile to note that in the proposed scheme the ML is applied on a reduced dimension symbol space, where the computations needed to detect the symbol is less. Since the remaining symbol space has been detected using column norm based ordering, the complexity of the system is reduced to a great extend.

\section{COMPLEXITY ANALYSIS}

The evaluation for the computational complexity of the proposed detection scheme is done with the number of real multiplications (NOMs) required to decode the transmitted complex symbols. The computational complexity for MMSE using Gauss Jordan elimination algorithm to compute matrix inverse is given by

$$
\left(\frac{56}{3}\right)\left(\frac{N}{2}\right)^{3}+40\left(\frac{N}{2}\right)^{2}+\left(\frac{34}{3}\right) \frac{N}{2}+1
$$

as cited in [13]. In the BSIDE algorithm, the QR decomposition of the $\mathrm{H}$ matrix requires $M N^{2}-\frac{N^{3}}{3}$ multiplications, to compute $Q^{H} Y, \tilde{d}$, and $\tilde{s}$, require $M^{2}$ multiplication and $\frac{N(N+3)}{2}$ multiplications and $\mathrm{N}$ divisions respectively. So the NOM required to perform up to step 2 is approximately given by

$$
M N^{2}-\frac{N^{3}}{3}+M^{2}+\frac{N(N+5)}{2}
$$

as per [14]. Let $U_{l}$ and $D_{l}$ be the NOMs required to compute $\left\{\theta\left(S_{l+1}^{[q]}\right)_{q=1}^{e_{l+1}}\right\}$ and $d_{l}$ at the $l^{t h}$ layer. Then the total NOMs for $N<l<\frac{N}{2}$ is given by

$$
\sum_{l=N}^{N}\left(\frac{C}{2}+C e_{l+1}+U_{l}+D_{l}\right)
$$

Where

$$
\begin{aligned}
& e_{l}=C^{N-l+1} \\
& U_{l}=(N-l) C^{N-l} \\
& D_{l}=N+\sum_{i=1}^{N} i
\end{aligned}
$$

The Maximum NOMs required for the proposed scheme can be expressed as

$$
\begin{aligned}
N O M_{\max }= & \left(\frac{56}{3}\right)\left(\frac{N}{2}\right)^{3}+40\left(\frac{N}{2}\right)^{2}+\left(\frac{34}{3}\right) \frac{N}{2}+1+. . \\
& . M N^{2}-\frac{N^{3}}{3}+M^{2}+\frac{N(N+5)}{2}+\sum_{l=N}^{\frac{N}{2}+1}\left(\frac{C}{2}+C e_{l+1}+U_{l}+D_{l}\right)
\end{aligned}
$$

The Minimum NOMs needed for the proposed scheme is given as

$$
N O M_{\min }=\frac{N(N+3 C-1)}{2}
$$

The minimum NOMs for conventional BSIDE remains the same as proposed, where as maximum NOMs varies. Thus the NOMs for the worst case are reduced.

\section{SIMULATION RESULTS}

Though ML detection is the optimum MIMO detection technique in the literature, its analysis is NP hard and hence the comparison for complexity is made with BSIDE algorithm. BSIDE is considered to be the alternate method with performance similar to ML and complexity less than SD. The reduction in computational complexity for the proposed scheme is achieved through combining the norm based ordering MMSE and BSIDE algorithm.

The upper layers of the tree having less number of child nodes, is detected with the help of BSIDE algorithm and lower layers, with more number of child nodes are traced by retaining the decision of MMSE detection, whose SNR is high and considered to be reliable. In this section we compare the proposed scheme with the existing BSIDE algorithm in terms of the performance and complexity.

Fig.4 shows the BER performance for $2 \times 2$ and $4 \times 4,4$ QAM MIMO systems. From the simulation result it is observed that the performance of the proposed scheme closely approach the BSIDE without any degradation. In the entire range of $E_{b} / N_{o}$ the BER performance remains the same for both BSIDE and proposed scheme. Whereas, in the complexity curve, there is a considerable improvement in the reduction of complexity for the proposed scheme until the $\mathrm{E}_{\mathrm{b}} / \mathrm{N}_{\mathrm{o}}$ reaches $25 \mathrm{~dB}$. Between $25 \mathrm{~dB}$ to $30 \mathrm{~dB}$ the BSIDE complexity slowly approaches the proposed and beyond $30 \mathrm{~dB}$ both the complexities become same. 


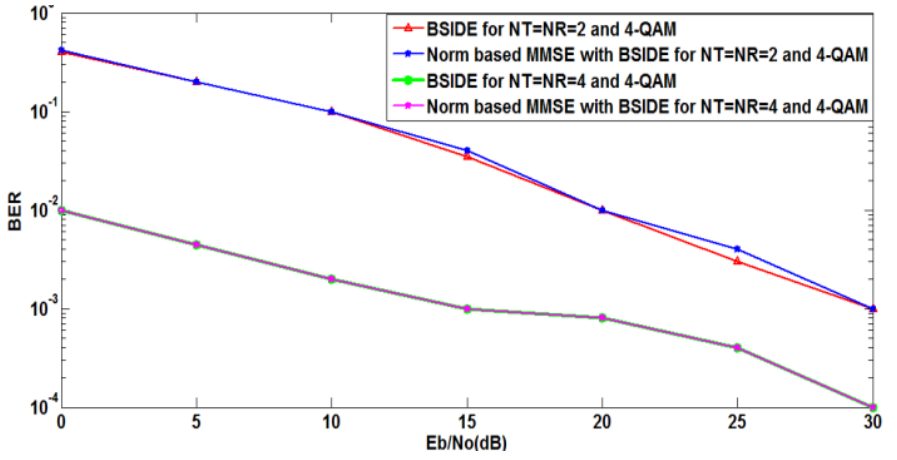

Fig.4. Performance comparison of 2x2 using 4-QAM and 4x4 MIMO using 4-QAM

It is clear from the simulation results that the proposed scheme works well for low and intermediate SNR signals. The complexity comparisons are given in Figures 5 and 6. The proposed scheme results in reduced complexity of $22 \%$ for $2 \times 2$ and $50 \%$ for $4 \times 4$ MIMO systems.

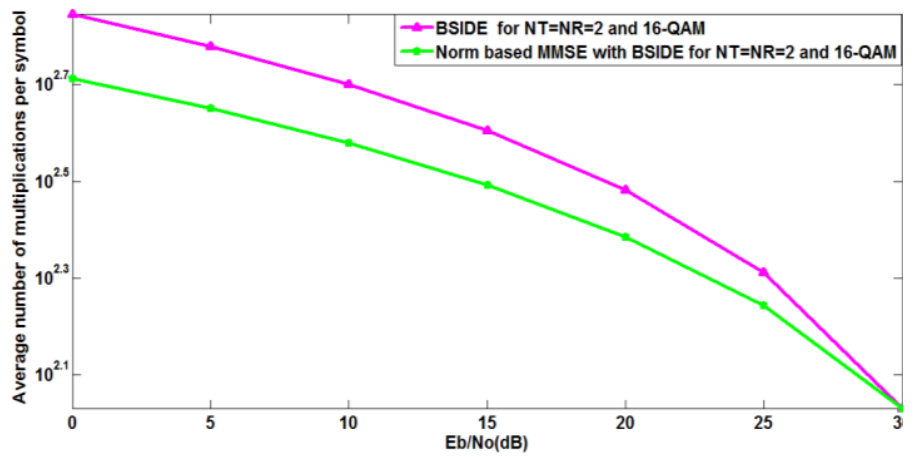

Fig.5. Complexity comparison of 2x2 MIMO using 16-QAM

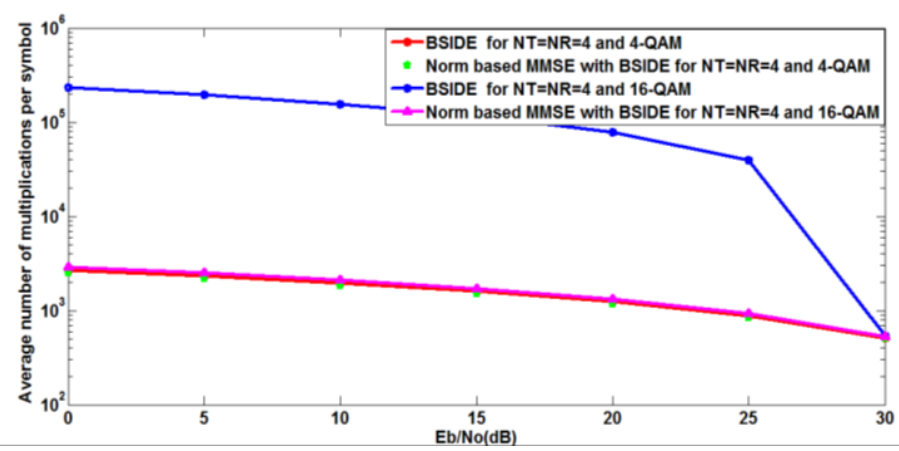

Fig.6. Complexity comparison of 4x4 MIMO using 4, 16- QAM

Table I. shows the complexity comparison for investigated combination of norm based MMSE with BSIDE and BSIDE algorithm. It is observed from the table that the maximum NOMs required, to decode a symbol for 2 × 2 4-QAM exceeds the BSIDE. The complexity comparisons are given in Figures 3 and 4 . The proposed scheme results in reduced complexity of $22 \%$ for $2 \times 216-\mathrm{QAM}$ and $98 \%$ for $4 \times 4$ 16-QAM MIMO system.

\section{CONCLUSION}

A hybrid scheme with reduced search space for MIMO detection has been proposed in this paper. The proposed scheme achieves less computational complexity and similar performance as Existing algorithm. This reduction in computational complexity is accomplished by exploiting the advantage of MMSE i.e, reliable detection over high SNR symbols, and concatenation of BSIDE algorithm for low SNR symbols.

TABLE I

COMPLEXITY COMPARISON FOR NORM BASED MMSE BSIDE DETECTION

\begin{tabular}{|c|c|c|c|c|c|c|c|c|c|c|}
\hline \multirow{4}{*}{ Category } & \multirow{4}{*}{ BER } & \multirow{4}{*}{$E_{b} / N_{0}$} & \multicolumn{8}{|c|}{ NOMs } \\
\hline & & & \multicolumn{4}{|c|}{$2 \times 2$} & \multicolumn{4}{|c|}{$4 \times 4$} \\
\hline & & & \multicolumn{2}{|c|}{ 4QAM } & \multicolumn{2}{|c|}{ 16QAM } & \multicolumn{2}{|c|}{ 4QAM } & \multicolumn{2}{|c|}{ 16QAM } \\
\hline & & & Max & Min & $\operatorname{Max}$ & Min & Max & Min & Max & Min \\
\hline \multirow{2}{*}{ BSIDE } & $10^{-4}$ & $30 \mathrm{~dB}$ & \multirow{2}{*}{191} & \multirow{2}{*}{95} & \multirow{2}{*}{699} & \multirow{2}{*}{107} & \multirow{2}{*}{2696} & \multirow{2}{*}{509} & \multirow{2}{*}{$2,33,775$} & \multirow[b]{2}{*}{533} \\
\hline & $10^{-2}$ & $15 \mathrm{~dB}$ & & & & & & & & \\
\hline \multirow{2}{*}{$\begin{array}{c}\text { Merge } \\
\text { Norm based } \\
\text { MMSE with } \\
\text { BSIDE }\end{array}$} & $10^{-4}$ & $30 \mathrm{~dB}$ & \multirow[b]{2}{*}{444} & \multirow[b]{2}{*}{95} & \multirow[b]{2}{*}{515} & \multirow[b]{2}{*}{107} & \multirow[b]{2}{*}{2530} & \multirow[b]{2}{*}{509} & \multirow[b]{2}{*}{2895} & \multirow[b]{2}{*}{533} \\
\hline & $10^{-2}$ & $15 \mathrm{~dB}$ & & & & & & & & \\
\hline $\begin{array}{c}\text { Reduction in } \\
\text { Complexity }\end{array}$ & - & - & & - & & $\%$ & & $\%$ & 98.5 & \\
\hline
\end{tabular}

\section{REFERENCES}

[1] P.W. Wolniansky, G.J. Foschini, G.D. Golden, and R.A.Valenzuela, "VBLAST: An architecture for realizing very high data rates over the richscattering wireless channel," Proc. URSI Int. Symp. Signals, Systems and Electronics, Pisa, Italy, pp. 295-300, 1998.

[2] C.-A, Shen, A. M. Eltawil, K. N. Salama, S. Mondal, "A Best-First Soft/Hard Decision Tree Searching MIMO Decoder for a 4x 4 64-QAM System", IEEE Transactions on Very Large Scale Integration (VLSI) systems, vol. 20 , no. 8 , pp. 1537-1541, 2012

[3] C. Qian, J. Wu, Y. R. Zheng, Z. Wang, "Two-Stage list sphere decoding for Under-Determined Multi-Input Multi-Output systems," IEEE Transactions on Wireless Communications, vol.12, no.12, pp.6476-6487, 2013.

[4] Y. Zhang,"Effective reduction for Sphere Decoder in linear multi-input multi-output channel systems", IET Communications, vol. 6, no. 11, pp. 1573-1578, 2012.

[5] L. Azzam, E. Ayanoglu, "Reduced complexity sphere decoding via a reordered lattice representation," IEEE Transactions on Communications, vol. 57, no. 9, pp. 2564-2569, September 2009.

[6] M. Mansour, S.P Alex, L.M.A Jalloul, "Reduced complexity soft output MIMO sphere detectors-part I: Algorithmic optimizations", IEEE Transactions on Signal Processing, vol. 62, no. 21, pp. 5505-5520, 2014.

[7] M. Wu, B. Yin, G. Wang, C. Dick, "Large scale MIMO detection for 3GPP LTE: Algorithms and FPGA implementations", IEEE Selected Topics in Signal Processing, vol. 8, no. 5, pp. 916-929, 2014.

[8] J Janhunen, O Silvén, M Juntti, "Programmable processor implementations of K-best list sphere detector for MIMO receiver,' Elsevier Journal of Signal Processing, vol. 90, pp. 313-323, 2009.

[9] S. Roger, A. Gonzalez, V. Almenar, A. M. Vidal, "Practical aspects of preprocessing techniques for K-Best tree search MIMO detectors", Elsevier Journal of Computers and Electrical Engineering, vol. 37, pp. 451-460, May 2011.

[10] G. Wang, H. Leib, "Sphere decoding for MIMO systems with newton iterative matrix inversion", IEEE Communications Letters, vol. 17, no. 2 , pp. 389-392, 2013.

[11] R. H. Chen, W.-H. Chung, "Reduced complexity MIMO detection scheme using statistical search space reduction", IEEE Communications Letters, vol. 16, no.3, pp.292-295, 2012.

[12] R. Y. Chang, W.-H. Chung, "Low complexity MIMO detection based on post Equalization subspace search", IEEE Transactions on Vehicular Technology, vol. 61, no. 1, pp.375-380, January 2012.

[13] C.-Y. Hung, R. Y. Chang, W.-H. Chung, "A hybrid MMSE and K-Best detection scheme for MIMO systems", IEEE Vehicular Technology Conference (VTC), pp. 1-5, September 2012.

[14] HG Kang, I Song, J Oh, J Lee, S. Yoon, "Breadth-first signal decoder: A Novel maximum likelihood scheme for multi input multi output systems," IEEE Transactions on Vehicular Technology, vol. 57, pp.1576-1583, 2008 . 